\title{
AVALIAÇÃO DOS DANOS CAUSADOS POR FORMIGAS- CORTADEIRAS DO GÊNERO Acromyrmex (Hymenoptera) AOS PLANTIOS DE Pinus taeda NO PLANALTO SUL-CATARINENSE
}

\author{
David Alexandre Buratto ${ }^{1}$, Josiane Teresinha Cardoso ${ }^{2}$, Flávio Augusto Rolim ${ }^{3}$, Wilson Reis Filho ${ }^{4}$ \\ ${ }^{1}$ Eng. Florestal, Mestrando em Engenharia Florestal, UFPR, Curitiba, PR, Brasil - davidburatto@ yahoo.com.br \\ ${ }^{2}$ Bióloga, Dra ., Depto. Engenharia Ambiental, UDESC, Lages, SC, Brasil - jositc@yahoo.com.br \\ ${ }^{3}$ Eng. Florestal, M.Sc., Ponte Alta do Norte, SC, Brasil - flavio.rolim@gerdau.com.br \\ ${ }^{4}$ Eng. Agrônomo, Dr., EPAGRI, Curitiba, PR, Brasil - wilson@cnpf.embrapa.br \\ Recebido para publicação: 14/09/2011 - Aceito para publicação: 20/12/2012
}

\begin{abstract}
Resumo
As formigas cortadeiras pertencentes ao gênero Acromyrmex (Hymenoptera: Formicidae), representam um dos principais problemas do empreendimento florestal no Brasil. Estudos objetivando determinar a ocorrência e os danos causados por essas formigas em áreas de plantio de Pinus na região Sul do Brasil são escassos. Os principais objetivos desta pesquisa foram a determinação da densidade de ninhos em áreas de plantio de Pinus taeda L. com e sem controle químico e a quantificação de plantas atacadas. Os estudos foram conduzidos no período de janeiro a junho de 2010. O experimento foi instalado em uma fazenda situada no município de Anita Garibaldi (SC). Foram demarcadas quatro parcelas de 0,5 ha, sendo que duas receberam um combate pré-plantio com isca formicida e duas não. Após o plantio, foram realizados censos quinzenais em cada parcela. Constatou-se a ocorrência de formigas das espécies Acromyrmex crassispinus Forel 1909 e Acromyrmex heyeri Forel 1899. Os danos totais médios por hectare causados às mudas foram no máximo de $3,0 \%$ no $35^{\circ}$ dia e de $5,38 \%$ no $63^{\circ}$ dia de avaliação. Os controles às formigas podem ser diminuídos se forem levados em consideração época do plantio, densidade dos formigueiros e porcentagem de danos causados às mudas.

Palavras-chave: Controle químico; formigas; isca formicida.
\end{abstract}

\begin{abstract}
Assessment of damages caused by leaf-cutting ants of genus Acromyrmex (Hymenoptera: Formicidae) in plantations of Pinus taeda in Santa Catarina southern highlands. Leaf-cutting ants of genus Acromyrmex (Hymenoptera: Formicidae) represent one of the main problems of forest enterprise in Brazil. Researches aiming to determine occurrence and damage caused by such ants in areas of pine plantation in southern Brazil are scarce. The main aims of this research were determining density of nests in Pinus taeda L. planted areas with and without chemical control, and quantification of attacked plants. The research was developed from January to June 2010. The experiment was set up on a farm in the municipality of Anita Garibaldi, SC. Four plots of 0.5 ha were marked out, two received a pre-planting to combat ant bait, and two others not. After planting, censuses had been managed biweekly to each plot. It revealed the occurrence of the following ant species: Acromyrmex crassispinus Forel 1909 and Acromyrmex heyeri Forel 1899. The average total damage caused to seedlings per hectare reached the maximum of $3.0 \%$ at the $35^{\text {th }}$ day, and $5.38 \%$ at the $63^{\text {rd }}$ day of evaluation. Ants control can be decreased if it is considered planting time, density of nests and seedlings damage percentage.

Keywords: Ant; ant bait; chemical control.
\end{abstract}

\section{INTRODUÇÃO}

O Brasil possui uma das maiores áreas de florestas plantadas do mundo, sendo um dos maiores produtores mundiais de madeira, carvão e celulose a partir de maciços florestais homogêneos. Os cultivos florestais se destacam por oferecer importantes contribuições nos aspectos econômicos, sociais e ambientais, uma vez que servem como fontes de matéria-prima, ajudando a diminuir a pressão sobre as 
florestas nativas (ASSOCIAÇÃO BRASILEIRA DE PRODUTORES DE FLORESTAS PLANTADAS (ABRAF), 2010).

Espécies dos gêneros Pinus e Eucalyptus são as mais utilizadas em reflorestamentos no Sul e Sudeste do país. Os plantios de Pinus ocupam mais de um milhão e meio de hectares, abrangendo os estados do Paraná $(39,1 \%)$, Santa Catarina (31,1\%), Rio Grande do Sul $(9,6 \%)$, Minas Gerais $(7,8 \%)$ e São Paulo $(9,2 \%)$ (ABRAF, 2011). Os estados da região Sul são os grandes produtores nacionais de matérias-primas provenientes dessas árvores e se destacam por apresentarem condições propícias para o desenvolvimento e a alta produtividade das espécies de Pinus (HOEFLICH, 2004).

Dentre as diversas espécies de pínus adaptadas à região Sul, destaca-se o Pinus taeda L., que representa aproximadamente $80 \%$ das florestas plantadas, principalmente nos estados de Santa Catarina e Paraná (SOCIEDADE BRASILEIRA DE SILVICULTURA (SBS), 2006), com um aumento considerável na aquisição de novas áreas para reflorestamento pelas empresas do setor (HOEFLICH, 2004).

Os prejuízos causados pelos insetos considerados pragas aos povoamentos florestais no Brasil têm levado as empresas do setor a investir recursos financeiros e científicos com o intuito de minimizar os danos causados aos reflorestamentos. Dentre as principais pragas florestais, ressaltam as formigascortadeiras, que alcançaram o nível de pragas devido aos grandes prejuízos que causam aos povoamentos de Pinus e Eucalyptus.

As formigas-cortadeiras pertencem ao gênero Acromyrmex, também conhecidas como quenquéns, e ao gênero Atta, comumente denominadas de saúvas. Os danos causados pelas formigascortadeiras provêm do corte de partes vegetativas para a utilização como substrato no cultivo do fungo usado na sua alimentação. Os ataques às plantas ocorrem preferencialmente nas fases de pré-corte, pósplantio e início da condução da brotação (BOARETTO; FORTI, 1997; ANJOS et al., 1998; NICKELE, 2008).

O gênero Acromyrmex conta com 63 espécies, das quais 28 têm ocorrência constatada no Brasil (MAYHÉ-NUNES, 1991). Já do gênero Atta, ocorrem no Brasil 10 espécies e três subespécies (DELLA LUCIA et al., 1993). As espécies de Acromyrmex são reconhecidas por apresentarem de quatro a cinco pares de espinhos na parte dorsal do tórax, os quais podem ser muito reduzidos no pronoto de algumas espécies; já as saúvas possuem somente três pares (MAYHÉ-NUNES, 1991).

A espécie Acromyrmex crassispinus Forel 1909 distribui-se na Argentina, Brasil, Paraguai e Uruguai (HÖLLDOBLER; WILSON, 1990; MAYHÉ-NUNES, 1991). No Brasil, ocorrem em São Paulo, Rio de Janeiro, Paraná, Santa Catarina, Rio Grande do Sul, Distrito Federal e Minas Gerais (GONÇALVES, 1961; MAYHÉ-NUNES, 1991; DELLA LUCIA et al., 1993), sendo a espécie de formiga-cortadeira mais comum na região Sul do Brasil (GONÇALVES, 1961; DELLA LUCIA et al., 1993; RANDO; FORTI, 2005).

Pouco se conhece sobre a distribuição e comportamento das espécies de formigas-cortadeiras em áreas de Pinus no Sul do Brasil. Isso indica que os controles realizados nessa região são baseados em conhecimento sobre a ação de espécies de formigas-cortadeiras de outras partes do Brasil, fato que muitas vezes leva a excessos nas doses de isca. Em função disso, torna-se necessário identificar as espécies predominantes, densidade de formigueiros e porcentagem de danos causados às mudas plantadas no Sul do país, a fim de se aperfeiçoarem os métodos de controle.

Questões econômicas e ambientais têm obrigado as empresas do setor florestal a melhorarem o método de controle químico e incentivado o conhecimento acerca de novos princípios ativos e metodologias para o controle de formigas-cortadeiras. Alguns métodos alternativos de controle têm sido constantemente mencionados, como, por exemplo: controle cultural, mecânico e biológico, uso de plantas resistentes e tóxicas e uso de feromônios. Contudo, até o momento, o controle químico baseado no emprego de iscas granuladas e utilização de inseticida na formulação pó seco são os únicos possíveis de serem usados em grande escala (ARAÚJO et al., 2003; NICKELE, 2008).

Sendo assim, o presente projeto teve como objetivo identificar as espécies de formigascortadeiras em área de plantio de $P$. taeda em campo nativo no planalto sul-catarinense e determinar a quantidade de ninhos e de plantas atacadas por essas formigas.

\section{MATERIAL E MÉTODOS}

O estudo foi conduzido na Fazenda Pinheiros Ralos, em plantios recentes de P. taeda em região 
de campo nativo localizados no município de Anita Garibaldi (SC), pertencentes à Florestal Rio Marombas Ltda. Esse município está localizado na região bioclimática I de Santa Catarina, no Planalto Sul-Catarinense, com altitude variando de 600 a 1.300 metros, vegetação do tipo Floresta Ombrófila Mista, temperatura média anual de 12 a $19{ }^{\circ} \mathrm{C}$ e precipitação anual de 1.300 a $2.400 \mathrm{~mm}$, sem déficit hídrico (EMPRESA BRASILEIRA DE PESQUISA AGROPECUÁRIA (EMBRAPA), 1988).

Foram instaladas linhas de plantio no terreno, as quais foram feitas de forma mecanizada com o auxílio de rippel e de forma manual em áreas rochosas e muito acidentadas. Foram instaladas em janeiro de 2010 duas parcelas para o Tratamento 1 (com controle de formiga) de $5.000 \mathrm{~m}^{2}$, com dimensões de 100 por $50 \mathrm{~m}$ entre linhas de plantio, e duas parcelas com as mesmas dimensões para o Tratamento 2 , sem controle de formigas. Todas as parcelas foram demarcadas com o auxílio de um piquete de madeira de $1,5 \mathrm{~m}$ de comprimento, alocado em cada vértice, conforme figura 1 . Foram feitas bordas de aproximadamente 10 metros entre parcelas e de 500 metros entre tratamentos. Tratamento 1: $\mathrm{F}=$ Fazenda; $\mathrm{PR}=$ Pinheiros Ralos; $\mathrm{P}=$ Parcela; $9,10,11$ e $12=$ números das parcelas $; \mathrm{C}$ ou $\mathrm{S}=$ com ou sem controle; corresponde às parcelas FPRP9C e FPRP10C. Tratamento 2: sem controle, representado pelas parcelas FPRP11S e FPRP12S.

As mudas foram plantadas no mês de abril de 2010, contendo em cada parcela 20 linhas e 21 entrelinhas de plantio, com 40 plantas/linha, gerando uma densidade de 1.600 mudas/ha.

O controle de formigas pela empresa é realizado de forma sistemática e localizado antes e após o plantio, sendo essa etapa denominada de controle pós-plantio, o qual pode ser composto de até cinco controles dependendo da situação morfovisual e da intensidade dos danos causados às mudas plantadas no decorrer dos primeiros anos. Antes do plantio, pode ser realizado de um a dois controles, num intervalo de 30 dias, com intuito de eliminar qualquer vestígio de formigas na região. Em todos os controles é utilizada ISCA FORMICIDA ATTA MEX-S 50x 500gr, cujo princípio ativo é a sulfluramida $(3 \mathrm{~g} / \mathrm{kg})$. A manutenção da vegetação presente na área é realizada com roçadas ao longo das linhas de plantio e roçada $100 \%$, que inclui linhas e entrelinhas de plantio.

As avaliações na área experimental foram realizadas em forma de censos quinzenais com início em abril e término em maio de 2010, totalizando seis avaliações.

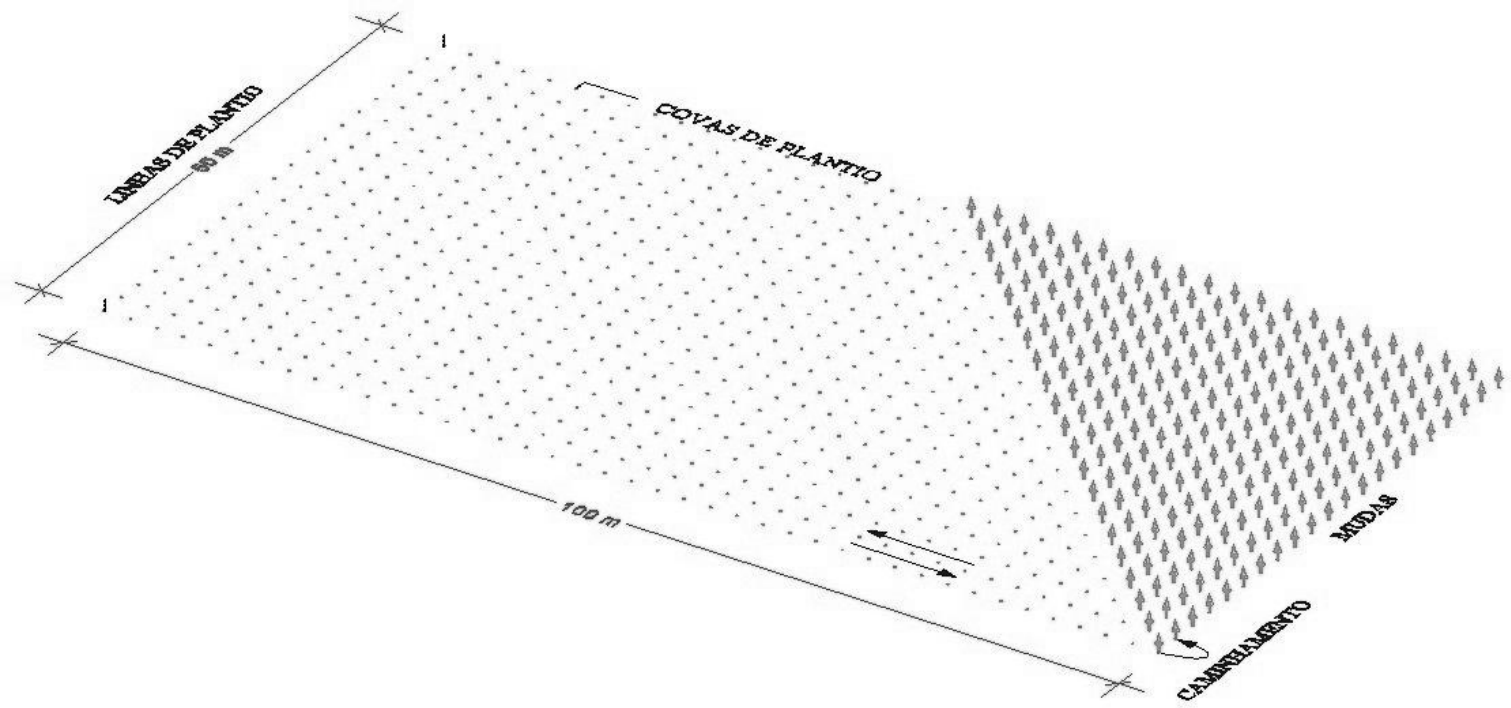

Figura 1. Croqui do modelo das parcelas instaladas nas áreas experimentais de cada fazenda.

Figure 1. Sketch of the model installed in the areas of experimental plots of each farm.

Realizaram-se censos quinzenais dentro de cada parcela, a fim de quantificar a densidade dos ninhos em cada uma delas, registrando-se o surgimento e permanência de cada formigueiro.

Os formigueiros encontrados foram demarcados com o auxílio de um piquete de madeira de $1,5 \mathrm{~m}$ de comprimento. $\mathrm{Na}$ extremidade de cada piquete, foi amarrada uma fita amarela de aproximadamente $1,0 \mathrm{~m}$ de comprimento por $5 \mathrm{~cm}$ de largura, sendo que cada um recebeu um número 
sequencial, com a finalidade de facilitar o trabalho subsequente dos mesmos (NICKELE, 2008).

Os dados coletados foram analisados e interpretados numericamente. Aplicou-se teste de Tukey nos valores médios obtidos com intervalo de confiança $(\mathrm{p}<0,05)$, utilizando-se o aplicativo Computacional Statistica versão 5.5.

Coletaram-se 10 indivíduos de cada formigueiro para a identificação da espécie, dando-se preferência às operárias máximas, ou seja, as maiores. As formigas foram acondicionadas em frascos contendo álcool $70 \%$ e posteriormente montadas e identificadas. Para a identificação da espécie em estudo, alguns exemplares foram encaminhados ao Laboratório de Entomologia da Empresa de Pesquisas Agropecuárias - EMBRAPA Florestas.

Os danos causados às mudas foram classificados usando-se a metodologia descrita por Nickele (2008), que se baseou em quatro níveis de desfolha provocados pelas formigas-cortadeiras em $P$. taeda recém-plantado, sendo: nível $1=50 \%$ de desfolha; nível $2=75 \%$ de desfolha; nível $3=100 \%$ de desfolha; nível $4=100 \%$ de desfolha incluindo o corte do meristema apical. As plantas danificadas foram contabilizadas em cada avaliação e não se repetiu em observações subsequentes o registro das mudas atacadas nas avaliações anteriores.

\section{RESULTADOS E DISCUSSÃO}

Constatou-se na área de estudo a ocorrência de formigas-cortadeiras do gênero Acromyrmex (quenquém), tendo a predominância da espécie Acromyrmex crassispinus Forel 1909 e Acromyrmex heyeri Forel 1899.

Acromyrmex crassispinus é a espécie de formiga cortadeira mais comum do Sul do Brasil, conhecida popularmente como "quenquém-de-cisco", devido ao hábito de construir ninhos com montes de ciscos (DELLA LUCIA et al., 1993; GONÇALVES, 1961; MAYHÉ-NUNES, 1991).

Em toda a área experimental, não foram encontradas formigas do gênero Atta (saúvas), porém foi observada a presença dessa espécie em estradas da fazenda.

Averiguou-se um número total de 28 formigueiros em todas as parcelas, no entanto apenas os formigueiros das parcelas sem controle de formigas mantiveram-se ativos durantes todos os meses de pesquisa, constatando-se assim a eficiência do controle, que acabou por eliminar os ninhos das parcelas controladas (Tabela 1).

Tabela 1. Número e média por hectare de formigueiros encontrados em cada parcela na Fazenda Pinheiros Ralos, Anita Garibaldi, SC.

Table 1. Number and average per hectare of nests found in each plot in Pinheiros Ralos farm, Anita Garibaldi, SC.

\begin{tabular}{lccccccccc}
\hline \multirow{2}{*}{ Censo } & \multirow{2}{*}{ Mês } & \multicolumn{2}{c}{ FPRP9C } & \multicolumn{2}{c}{ FPRP10C } & \multicolumn{2}{c}{ FPRP11S } & \multicolumn{2}{c}{ FPRP12S } \\
\cline { 3 - 10 } & & $\sum$ & Média/ha & $\sum$ & Média/ha & $\sum$ & Média/ha & $\sum$ & Média/ha \\
\hline $1^{\circ}$ & Fev. & 14 & 28,0 & 8 & 16,0 & 5 & 10,0 & 1 & 2,0 \\
$2^{\circ}$ & Mar. & 0 & 0,0 & 0 & 0,0 & 0 & 0,0 & 1 & 2,0 \\
$3^{\circ}$ & Mar. & 0 & 0,0 & 2 & 4,0 & 0 & 0,0 & 2 & 4,0 \\
$4^{\circ}$ & Abr. & 0 & 0,0 & 1 & 2,0 & 1 & 2,0 & 2 & 4,0 \\
$5^{\circ}$ & Abr. & 0 & 0,0 & 1 & 2,0 & 1 & 2,0 & 1 & 2,0 \\
$6^{\circ}$ & Maio & 0 & 0,0 & 1 & 2,0 & 1 & 2,0 & 1 & 2,0 \\
$7^{\circ}$ & Maio & 0 & 0,0 & 0 & 0,0 & 1 & 2,0 & 1 & 2,0 \\
$8^{\circ}$ & Jun. & 0 & 0,0 & 0 & 0,0 & 1 & 2,0 & 1 & 2,0 \\
\hline
\end{tabular}

FPRP9C: Fazenda Pinheiros Ralos, parcela 9, com controle de formigas; FPRP10C: Fazenda Pinheiros Ralos, parcela 10, com controle de formigas; FPRP11S: Fazenda Pinheiros Ralos, parcela 11, sem controle de formigas; FPRP12S: Fazenda Pinheiros Ralos, parcela 12, sem controle de formigas.

Foi constatado que o ataque ocorreu somente nas parcelas sem controle de formigas-cortadeiras (FPRP11S e FPRP12S), observando-se que o ataque mais intenso foi 35 dias após o plantio (Tabela 2), somando 22 plantas atacadas durante a quinta avaliação. Percebeu-se também que a maior incidência de ataque das formigas foi no nível 3, sendo cortadas todas as acículas das mudas, ficando somente o meristema apical. 
Littledyke e Cherret (1978) citam que as formigas-cortadeiras têm preferências significativas por folhas mais novas, o que foi confirmado por Nickele (2008), que verificou que a espécie A. crassispinus apresentou preferências pelas acículas mais jovens de plantas de pínus. Segundo Hubbell e Wiemer (1983), o ataque de formigas-cortadeiras acontece preferencialmente às folhas novas, porque as folhas maduras de um mesmo vegetal podem conter substâncias repelentes ou tóxicas a herbívoros. Cantarelli $e t$ al. (2003), ao avaliarem os óleos essenciais de acículas jovens, quando comparadas com acículas mais velhas de $P$. taeda, verificaram que compostos monoterpênicos, tais como $\alpha$-pineno, $\beta$-pineno, limoneno e mirceno, foram encontrados somente nas acículas mais velhas da planta. Os demais compostos, principalmente sesquiterpenos, foram encontrados tanto em acículas novas como em acículas velhas, no entanto com algumas diferenças de concentração. Essas substâncias, observadas somente nas acículas mais velhas, podem estar influenciando na escolha do corte pelas formigas.

A quantidade de mudas de $P$. taeda danificadas está presente na tabela 2 , onde se tem a porcentagem média de plantas atacadas por hectare em cada um dos diferentes níveis de desfolha, a média de danos 35 dias após o plantio e ao final de todas as avaliações.

Tabela 2. Soma das mudas de P. taeda danificadas por formigas cortadeiras na fazenda Pinheiros Ralos, Anita Garibaldi, SC.

Table 2. Sum of seedlings of P. taeda damaged by leaf-cutting ants in Pinheiros Ralos farm, Anita Garibaldi, SC.

\begin{tabular}{|c|c|c|c|c|c|c|c|c|c|c|}
\hline \multirow{2}{*}{ Avaliação } & \multirow{2}{*}{$\begin{array}{c}\text { Dias após o } \\
\text { plantio }\end{array}$} & \multicolumn{4}{|c|}{ Com controle } & \multicolumn{4}{|c|}{ Sem controle } & \multirow{2}{*}{ Soma } \\
\hline & & N1 & N2 & N3 & $\mathrm{N} 4$ & N1 & $\mathrm{N} 2$ & N3 & $\mathrm{N} 4$ & \\
\hline $1^{\mathrm{a}}$ avaliação & 7 dias & 0,0 & 0,0 & 0,0 & 0,0 & 1,0 & 1,0 & 1,0 & 0,0 & 3,0 \\
\hline $2^{\mathrm{a}}$ avaliação & 14 dias & 0,0 & 0,0 & 0,0 & 0,0 & 0,0 & 4,0 & 0,0 & 0,0 & 4,0 \\
\hline $3^{\mathrm{a}}$ avaliação & 21 dias & 0,0 & 0,0 & 0,0 & 0,0 & 0,0 & 0,0 & 4,0 & 0,0 & 4,0 \\
\hline $4^{\mathrm{a}}$ avaliação & 28 dias & 0,0 & 0,0 & 0,0 & 0,0 & 0,0 & 0,0 & 13,0 & 2,0 & 15,0 \\
\hline $5^{\mathrm{a}}$ avaliação & 35 dias & 0,0 & 0,0 & 0,0 & 0,0 & 0,0 & 8,0 & 14,0 & 0,0 & 22,0 \\
\hline Média/ha & 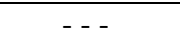 & 0,0 & 0,0 & 0,0 & 0,0 & 1,0 & 13,0 & 32,0 & 2,0 & 48,0 \\
\hline$\%$ Média de danos/ha & --- & 0,0 & 0,0 & 0,0 & 0,0 & 0,1 & 0,8 & 2,0 & 0,1 & 3,00 \\
\hline $6^{\mathrm{a}}$ avaliação & 42 dias & 0,0 & 0,0 & 0,0 & 0,0 & 0,0 & 8,0 & 8,0 & 1,0 & 17,0 \\
\hline $7^{\mathrm{a}}$ avaliação & 49 dias & 0,0 & 0,0 & 0,0 & 0,0 & 0,0 & 0,0 & 0,0 & 0,0 & 0,0 \\
\hline $8^{\mathrm{a}}$ avaliação & 56 dias & 0,0 & 0,0 & 0,0 & 0,0 & 0,0 & 0,0 & 5,0 & 0,0 & 5,0 \\
\hline $9^{\mathrm{a}}$ avaliação & 63 dias & 0,0 & 0,0 & 0,0 & 0,0 & 0,0 & 2,0 & 12,0 & 2,0 & 16,0 \\
\hline Média/ha & $-\ldots$ & 0,0 & 0,0 & 0,0 & 0,0 & 1,0 & 23,0 & 57,0 & 5,0 & 86,0 \\
\hline & & & & & & $\mathrm{a}$ & $a b$ & $\mathrm{~b}$ & $\mathrm{a}$ & \\
\hline$\%$ Média de danos /ha & $-\ldots$ & 0,0 & 0,0 & 0,0 & 0,0 & 0,06 & 1,44 & 3,56 & 0,31 & 5,38 \\
\hline
\end{tabular}

N1: nível 1 de dano (50\% de desfolha); N2: nível 2 de dano (75\% de desfolha); N3: nível 3 de dano (100\% de desfolha); N4: nível 4 de dano ( $100 \%$ de desfolha incluindo o corte do meristema apical).

Segundo o teste de Tukey, não houve diferença estatística entre os tratamentos com e sem controle de formigas. Uma vez que houve apenas o controle pré-plantio na área de estudo, é necessária uma reavaliação do uso da quantidade de formicida por hectare usado durante a realização do controle, já que, mesmo quando este não é feito, verifica-se que os danos não são significativos para o produtor.

O nível 1 de dano (50\% de desfolha) foi o que representou menor valor de ataque nas mudas, com uma porcentagem média de $0,06 \%$ de plantas danificadas, sendo esse dano contabilizado somente na primeira avaliação.

O valor médio total de plantas atacadas por formigas em todos os níveis de desfolha durante todo o período de avaliação (63 dias) foi de 86 mudas por hectare (Figura 2). Esse valor corresponde a uma porcentagem média total de plantas danificadas de $5,38 \%$ por hectare (Tabela 2).

A dosagem de formicida utilizado pelas empresas da região pode não ser justificável, devido à baixa quantidade de formigueiros encontrados e pela baixa taxa de danos ocasionada nas plantas. Comumente, 30 dias após o plantio as empresas da região realizam o replante das mudas quando as áreas plantadas alcançam uma porcentagem mínima de 5\% de perda, que podem ser causadas por diversos fatores, como, por exemplo, intempéries climáticas, ataques de animais, ações antrópicas etc. Sendo assim, observou-se no presente estudo que 35 dias após o plantio a porcentagem média de danos por 
hectare foi de $3 \%$, condições sob as quais o controle de formigas-cortadeiras pode ser reduzido ou até mesmo eliminado.

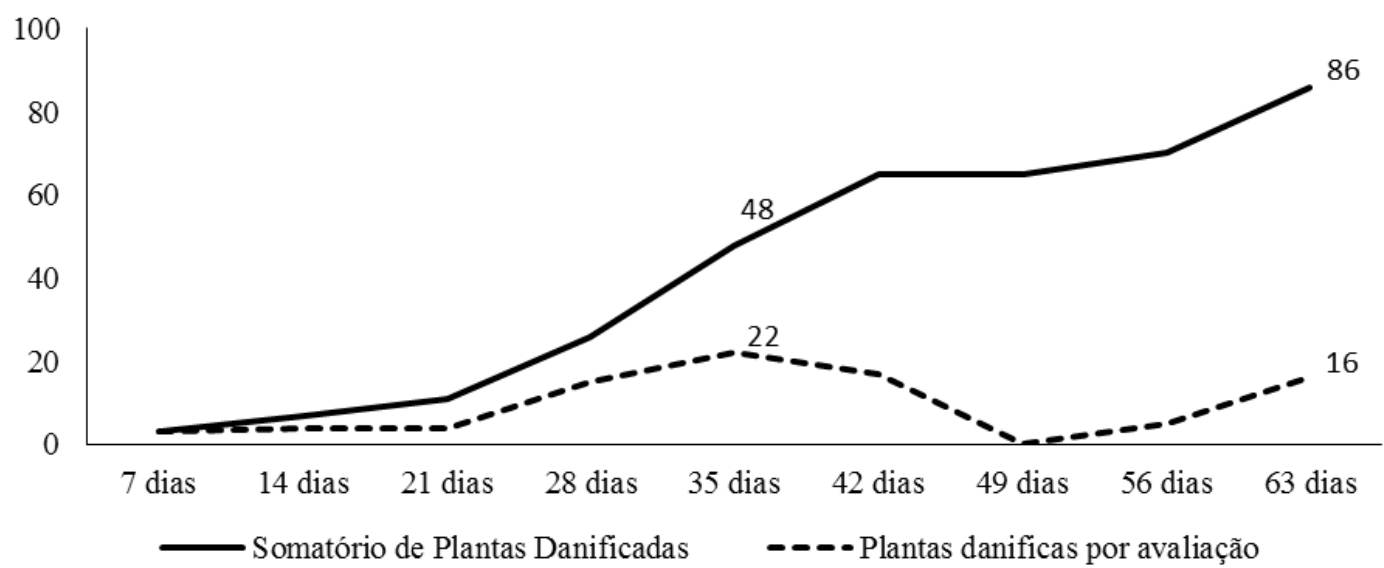

Figura 2. Soma dos danos causados às mudas de $P$. taeda por formigas-cortadeiras durante o período de 63 dias de avaliação.

Figure 2. Sum of damage to seedlings of P. taeda by leaf-cutting ants during the 63 days of evaluation. Eixo x: dias; eixo y: número de plantas danificadas.

A quantidade de mudas danificadas por hectare pode ser reduzido se for levado em consideração que os danos causados às mudas está diretamente relacionado com o período do ano em que ocorre o plantio, optando-se por épocas em que a atividade de formigas-cortadeiras é modificada, devido a fatores climáticos e nível de desenvolvimento dos ninhos. Lima et al. (2001) verificaram que as formigascortadeiras, normalmente, são muito ativas durante a noite, mas, em locais sombreados e durante períodos frios, a atividade de forrageamento e corte ocorre durante o dia. As iscas formicidas podem ainda se deteriorar com maior facilidade em virtude das intempéries climáticas a que ficam submetidas em campo em cada época do ano (por exemplo: umidade, insolação, ressecamento etc.).

Segundo Reis Filho (2010) (informação verbal), a quantidade de formicida utilizada no controle de formigas, segundo protocolos vigentes, é alta em relação à quantidade necessária para promover a mortalidade dos insetos no interior dos ninhos. Uma pequena quantidade de formicida é carregada para o interior do formigueiro, causando a eliminação das jardineiras, responsáveis pela alimentação da colônia.

O sucesso do controle formicida não está relacionado necessariamente à quantidade de isca aplicada nas áreas, podendo essa ser aperfeiçoada, devido à baixa densidade de formigueiros presentes, dos danos causados e das espécies de formigas predominantes na local.

\section{CONCLUSÃO}

- As espécies de formigas predominantes nas áreas experimentais foram Acromyrmex crassispinus e Acromyrmex heyeri.

- Os danos totais médios por hectare causados às mudas de P. taeda na Fazenda Pinheiros Ralos foi no máximo de 3,0\% 35 dias após o plantio e de 5,38\% 63 dias após ser efetuado o plantio.

- O controle das formigas-cortadeiras e a quantidade de isca utilizada em determinadas regiões podem ser diminuídos se forem levados em consideração os períodos do ano e a região em que vai ser efetuado o plantio, a densidade dos formigueiros e a porcentagem de danos causados às mudas.

- A otimização no uso de iscas formicidas pelas empresas florestais pode gerar ganhos econômicos e diminuir os impactos ambientais por elas causados.

\section{AGRADECIMENTOS}

À Universidade do Estado de Santa Catarina (UDESC /CAV); à Florestal Rio Marombas, pelo 
apoio financeiro e cessão da Fazenda para instalação do experimento; à Embrapa Florestas, pela parceria científica firmada para o sucesso deste trabalho.

\section{REFERÊNCIAS}

ANJOS, N. S.; DELLA-LUCIA, T. M. C.; MAYHÉ-NUNES, A. J. Guia prático sobre formigas cortadeiras em reflorestamentos. Ponte Nova: Graff Cor, 1998. 100 p.

ANUÁRIO ESTATÍSTICO DA ASSOCIAÇÃO BRASILEIRA DE PRODUTORES DE FLORESTAS PLANTADAS (ABRAF), Ano base: 2009. Brasília: 2010. 140 p.

. Ano base: 2010. Brasília: 2011. 130 p.

ARAÚJO, M. da S.; DELLA-LUCIA, T. M. C.; SOUZA, D. J. Estratégias alternativas de controle de formigas-cortadeiras. Bahia Agrícola, Salvador, v. 6, n. 1, p. 71 - 74, 2003.

BOARETTO, M. A. C.; FORTI, L. C. Perspectivas no controle de formigas-cortadeiras. Série Técnica IPEF, São Paulo, v. 11, n. 30, p. 31 - 46, 1997.

CANTARELli, E. B.; COSTA, E. C.; MOREL, A.; PEZZUTTI, R. V. Avaliação de óleos essenciais de acículas de Pinus taeda quanto à preferência no corte por formigas. In: SIMPÓSIO DE MIRMECOLOGIA, 16., 2003, Florianópolis. Anais... Florianópolis: UFSC, 2003. p. 477 - 479.

DELlA LUCIA, T. M. C.; VILELA, E. F. Métodos Atuais de Controle e Perspectivas. In: DELLA LUCIA, T. M. C. As formigas-cortadeiras. Viçosa: Folha de Viçosa, 1993. p. 163 - 190.

EMPRESA BRASILEIRA DE PESQUISAS AGROPECUÁRIA (EMBRAPA). Centro Nacional de Pesquisa de Florestas. Zoneamento ecológico para plantios no Estado de Santa Catarina. Curitiba, 1988. 113 p. (EMBRAPA - CNPF. Documentos, 21).

GONÇALVES, C. R. O gênero Acromyrmex no Brasil (Hymenoptera: Formicidae). Studia Entomologica, v. 4, n. 1-4, p. 113 - 180, 1961.

HOEFLICH, V. F. O papel das florestas para o desenvolvimento da sociedade brasileira. Revista da Madeira, v. 14, n. 83, p. 14 - 20, 2004.

HÖLLDOBLER, B.; WILSON, E. O. The ants. Cambridge: Harvard University Press, 1990. 733 p.

HUBBELL, S. P.; WIEMER, D. F. Host plant selection by Attini ant. In: JAISSON, P. Social insects in the tropics. Paris: University of Paris Press, 1983, v. 2, p. 133 - 157.

LIMA, C. A.; DELLA LUCIA, T. M. C.; SILVA, N. A. Formigas-cortadeiras: biologia e controle. Boletim de Extensão no 44. Viçosa, Minas Gerais, 2001. Universidade Federal de Viçosa. ISSN: 1234-5678.

LITTLEDYKE, M.; CHERRETT, J. M. Defence mechanisms in young and old leaves against cutting by the leaf-cutting ants, Atta cephalotes (L.) and Acromyrmex octospinosus (Reich) (Hymenoptera: Formicidae). Bulletin of Entomological Research, v. 68, n. 2, p. 263 - 271, 1978.

MAYHÉ-NUNES, A. J. Estudo de Acromyrmex (Hymenoptera: Formicidae) com ocorrência constatada no Brasil: subsídios para uma análise filogenética. 122 f. Dissertação (Mestrado em Entomologia) - Universidade Federal de Viçosa, Viçosa, MG, 1991.

NICKELE, M. A. Distribuição espacial, danos e planos de amostragem de Acromyrmex crassispinus (Forel, 1909) (Hymenoptera: Formicidae: Myrmicinae) em plantios de Pinus taeda L. (Pinaceae). 125 f. Dissertação (Mestrado em Ciências Biológicas) - Universidade Federal do Paraná, Curitiba, 2008.

RANDO, J. S. S.; FORTI, L. C. Ocorrência de formigas Acromyrmex Mayr, 1865, em alguns municípios do Brasil. Acta Scientiarum. Biological Sciences, v. 27, p. 129 - 133, 2005.

SOCIEDADE BRASILEIRA DE SILVICULTURA (SBS). Fatos e números do Brasil Florestal. São Paulo: SBS, 2006. 109 p.

STATISTICA for Windows 5.5. Computer program manual. Tulsa: StatSoft., 2000. 
